1239 The impact of ADHD and autism spectrum disorders on temperament, character, and personality development Henrik Anckarsäter, Ola Stahlberg, Tomas Larson, Catrin Hakansson, Sig-Britt Jutblad, Lena Niklasson,

Agneta Nydén, Elisabet Wentz, Stefan Westergren, C. Robert Cloninger, Christopher Gillberg, and Maria Rastam

I245 A validation of event-related fMRI comparisons between users of cocaine, nicotine, or cannabis and control subjects

Kevin Murphy, Veronica Dixon, Kathleen LaGrave, Jacqueline Kaufman, Robert Risinger, Alan Bloom, and Hugh Garavan

1252 Volumetric analysis and three dimensional glucose metabolic mapping of the striatum and thalamus in patients with autism spectrum disorders M. Mehmet Haznedar, Monte S. Buchsbaum, Erin A. Hazlett, Elizabeth M. LiCalzi, Charles Cartwright, and Eric Hollander
1264 Ferritin levels and their association with regional brain volumes in Tourette's syndrome

Daniel A. Gorman, Hongtu Zhu, George M. Anderson, Mark Davies, and Bradley S. Peterson

\section{NEW RESEARCH -} BRIEF REPORTS

1273 Prevalence of the metabolic syndrome among patients receiving clozapine

1. Steven Lamberti, David Olson,

John F. Crilly, Telva Olivares,

Geoffrey C.Williams, XinTu,WanTang,

Karen Wiener, Steven Dvorin,

and Marci B. Dietz

1276 Association of initial antipsychotic response to clozapine and long-term weight gain

Ya Mei Bai, Chao-Cheng Lin, Jen-Yeu Chen, Chih-Yuan Lin, Tung-Ping Su,

and Pesus Chou

1280 Suicidality in body dysmorphic disorder: a prospective study Katharine A. Phillips and William Menard
1282 Motor inhibition and cognitive flexibility in obsessive-compulsive disorder and trichotillomania Samuel R. Chamberlain, Naomi A. Fineberg, Andrew D. Blackwell, Trevor W. Robbins, and Barbara J. Sahakian

1285 Clinical assessment of pathological personality traits Drew Westen and Serra Muderrisoglu

1288 Effects of season of birth on autism spectrum disorders: fact or fiction? Alexander Kolevzon, Mark Weiser, Raz Gross, Gad Lubin, Haim Y. Knobler, James Schmeidler, Jeremy M. Silverman, and Abraham Reichenberg

1290 An MRI study of increased cortical thickness in autism

Antonio Y. Hardan, Sri Muddasani, Madhuri Vemulapalli, Matcheri S. Keshavan, and Nancy J. Minshew

The American Journal of Psychiatry is online at http: / /ajp. psychiatryonline.org 